\title{
Making Blind Robots See: The Synergy Between Fluorescent Dyes and Imaging Devices in Automated Proteomics
}

BioTechniques 28:944-957 (May 2000)

\author{
Wayne F. Patton \\ Molecular Probes, \\ Eugene, OR, USA
}

\section{INTRODUCTION}

An overall objective of biology is to clarify molecular mechanisms responsible for human disease to support the development of strategies for prophylactic and therapeutic intervention. Chronic and acute disease processes such as arteriosclerosis and inflammation usually manifest themselves at the level of protein activity (41). Arteriosclerosis is an example of a pathological process that occurs over a number of years, a period too long to precisely monitor transcription and translation. Both mRNA and protein are continually being synthesized and degraded, and thus final levels of protein are not easily obtainable by measuring mRNA levels $(41,42)$. On the other hand, certain biological responses, such as transient increases in blood vessel permeability accompanying inflammation, occur in seconds to minutes without transcription or translation changing the abundance of protein products $(22-24,41,60-62)$. Such processes typically rely on posttranslational modification of proteins, their subcellular redistribution and subsequent interaction with other proteins to produce a functional response $(16,41)$. The drug discovery industry is finding it increasingly necessary to incorporate proteomics into their repertoire of techniques to aid in transforming research discoveries into products for diagnosis, prognosis and therapy. After all, clinically relevant changes observed at the gene expression level are typically just a response to drug effects at the protein level (36).

Proteomics analysis provides data on when or if a predicted gene product is actually translated, the level and type of posttranslational modification it may undergo and its relative concentration compared with other gene products (27). After DNA is transcribed into mRNA, the exons may be spliced in different ways before being translated into proteins. Following the translation of mRNA by ribosomes, proteins are usually posttranslationally modified by the addition of different chemical moieties such as carbohydrate, lipid and phosphate groups, as well as through the physiologically relevant proteolytic cleavage of specific peptide bonds. These chemical modifications of proteins are crucial to modulating their function but are not directly coded for by genes. Considering such modifications, information from a single gene may culminate in the generation of 20 or more distinct protein species (41). Even though genomics provides comprehensive databases of sequence information, the sequencing of genomic DNA and spotting of cDNA onto chips does not address biological problems centered on pretranslational events and posttranslational modifications of proteins. DNA and mRNA provide no information concerning the activity of proteins, and though mRNA levels are often extrapolated to indicate the levels of expressed proteins, there is little correlation between the abundance of mRNA species and the actual amounts of proteins that they code for $(4,18)$.

A pivotal component of proteomics is its ability to accurately quantify vast numbers of proteins accurately and reproducibly. Although the primary aim of proteomics studies is to quantitatively define biological processes at the protein level, the commonly used stains for evaluating protein expression (such as Coomassie ${ }^{\circledR}$ Blue, colloidal gold and silver stain) do not provide the requisite dynamic range to be effective in 
this capacity. These stains are linear over a scant 10- to 40-fold range $(3,11,64)$. Fluorescence detection technologies provide a linear signal response relative to the amount of protein over a much wider range than is found for the nonfluorescent alternatives.

The abundance of individual proteins differs by as much as four orders of magnitude, and scarce components cannot be amplified by a PCR technique for proteins, as no such technique exists. Low abundance proteins, such as transcription factors and kinases that are present in 1-2000 copies per cell, often represent species that perform important regulatory functions $(10,21$,
26,29). The accurate detection of such low-abundance proteins is an important challenge to proteomics. Methods have recently been introduced to directly quantify the relative abundance of proteins in two different samples by mass spectrometry through covalent modification of cysteine residues from two cell populations with either hydrogenor deuterium-containing biotin affinity tags or by culturing different cell populations in ${ }^{15} \mathrm{~N}$ - and ${ }^{14} \mathrm{~N}$-enriched medium. However, the linear dynamic range of these methods has explicitly been demonstrated over only a four- to tenfold range $(18,38)$. Also, ion suppression phenomena associated with mass spectrometry prevent stoichiometric comparison of different proteins by such techniques $(30,65)$. Fluorescence detection methods currently offer the best solution to global protein quantitation in proteomics (48).

\section{AUTOMATION OF PROTEOMICS}

Obtaining information concerning global protein expression is technically difficult, and semiautomated procedures to accomplish this task are only beginning to be developed $(8,32-34,46)$. Typically, proteomics entails the simultaneous separation of proteins from a biological

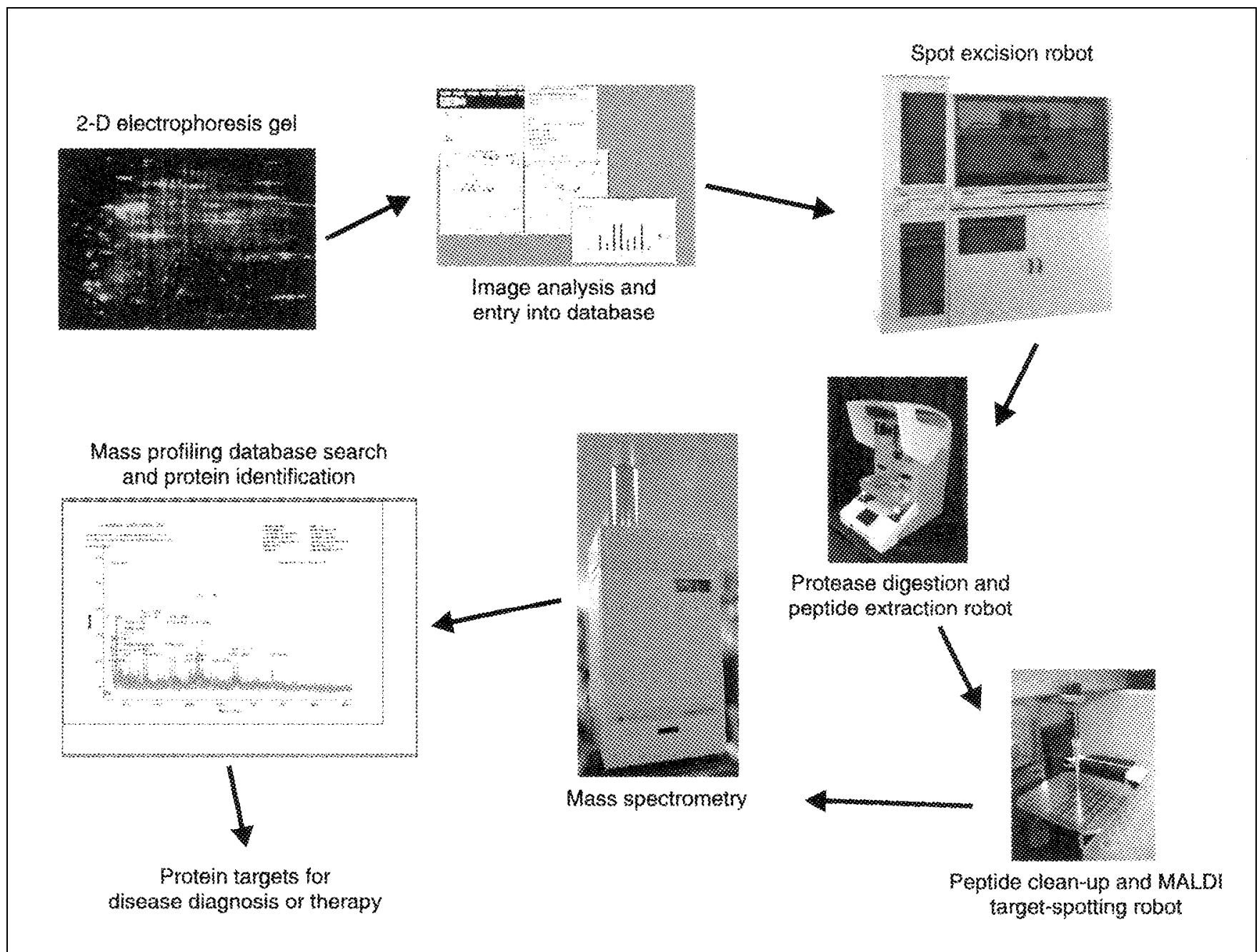

Figure 1. Flowchart of the technical steps required in semiautomated proteome analysis. Proteins resolved by 2-D gel electrophoresis are visualized by a CCD camera or laser scanner. Spot boundaries are detected, the amount of protein in each spot is established and their coordinates are determined. The images are then linked together into a gel database, and relevant spots are located by user-specified queries. Proteins of interest are excised from gels using a spot-picking robot interfaced to the imaging software. Spots are subsequently subjected to protease digestion and peptide extraction, and then peptide molecular weight is determined by mass spectrometry. The resulting peptide mass profile is then used in a database search to identify the protein. (Figure courtesy of Dr. Mary F. Lopez, Genomic Solutions, Chelmsford, MA, USA.) 
sample, the quantitation of the relative abundance of the proteins resolved during the separation and the identification of component species. Proteomics currently relies heavily on two-dimensional (2-D) gel electrophoresis, bioinformatics and mass spectrometry. Process throughput is being increased by using streamlined protein-staining technologies, spot-cutting robots that interface with analytical imaging devices and sample-handling systems that interface with mass spectrometers (34).

The technical steps ordinarily required for semiautomated proteomic analysis are illustrated in Figure 1. Here, 2-D gel electrophoresis (the separation of proteins in the first dimension according to their charge by isoelectric focusing and in the second dimension according to their relative mobility by SDS-PAGE) is used to simultaneously resolve thousands of proteins as constellation patterns of spots (39). A key development with respect to proteomics has been the ability to routinely run numerous high-resolution, largeformat, 2-D gels in a reproducible manner $(9,35,45)$.

After electrophoresis, proteins are visualized manually or by using a fully automated robotic staining system. Silver staining procedures are tedious and cumbersome, multistep processes that require careful timing $(47,48)$. Though robotic stainers can execute such protocols flawlessly, downstream compatibility with mass spectrometry requires the omission of glutaraldehyde from silver stain formulations, which compromises staining performance (51). Additional destaining steps are required to reduce background interference and suppression of signals often encountered in peptide mass-profiling experiments (15). Fluorescence procedures are generally much simpler to implement than silver staining methods. Covalent derivatiza- tion methods label proteins before electrophoresis, thus obviating the need for robotic stainers. Noncovalent methods stain proteins after electrophoresis using easy, one- to two-step protocols that can be performed manually or with automated stainers (37-39). Once proteins are visualized, an analytical imaging system combines machine vision, digital image processing and data processing to interpret the complex patterns generated by 2-D gel electrophoresis (40,43). After images are acquired, spot boundaries are detected, the amount of protein in each spot is determined and the coordinates of each spot are established. The images are then linked together in a gel database, and relevant spots are located by user-specified queries.

When proteins of interest have been identified, they are excised from gels manually or by using a fully automated system integrated with the gel analysis software (32-34). Automated systems 
consisting of a charge-coupled device (CCD) camera mounted on a robot arm are capable of excising 120-240 gel plugs per hour $(0.25-1.8 \mathrm{~mm}$ diameter), and an autoloader capacity permits several 2-D gels to be loaded for spot picking at one time. Spots are then automatically placed into 96 -well plates for delivery to a protein digestion workstation. Next, manual or automated protease digestion is performed, and the peptides generated are extracted from the gel matrix. Extracted peptides may then be automatically spotted onto a sample stage for delivery to the MALDI-TOF mass spectrometer. Mass spectrometry instruments fuel proteomics in the same way that highthroughput, fluorescence-based capillary electrophoresis DNA sequencers have propelled the genomic sequencing enterprise. Individual proteins are identified by comparing the actual masses of the peptide fragments generated from the proteins with the theoretical masses obtained from protein databases.

\section{LASER- AND CCD CAMERA- BASED IMAGING SYSTEMS}

A key component of any semiautomated, high-throughput proteomics environment is the instrumentation re- quired to detect the separated proteins and then to provide guidance to the spot excision robot in selecting targets for further characterization. In effect, this instrumentation makes the blind robot see! The most common instruments for obtaining images of electrophoresis gels incorporate a photomultiplier tube and laser scanner or a gas discharge transilluminator and CCD camera for protein visualization.

Laser scanners can detect fluorescently labeled or stained proteins in gels or on membranes with high sensitivity and resolution. Unlike conventional photography, a darkroom, film processor, chemicals and plumbing are not required to visualize fluorescent signals. Commonly used light sources in laser scanning devices include a diode laser $(635 \mathrm{~nm})$, helium-neon (He$\mathrm{Ne})$ laser $(633 \mathrm{~nm})$, argon-ion (Ar) laser $(514 \mathrm{~nm}, 488 \mathrm{~nm})$, frequency-doubled neodymium-yttrium-aluminumgarnet (Nd-YAG) laser (532 nm) and second harmonic generation (SHG) laser $(532 \mathrm{~nm}, 473 \mathrm{~nm})$. Two or more laser sources may be incorporated into commercial gel scanners, allowing a wider number of fluorophores to be used on the instruments. Solid-state laser scanners serially pass a beam of coherent light over each point of the sample in a rastering pattern by me- chanical or optical scanning, or a combination of the two methods (57).

A typical system uses a $20-\mathrm{mW}$ laser to excite fluorophores in the gel by mechanical movement of the optical unit across the sample in the Y-direction (Figure 2). The incident light from the laser beam is directed onto the sample in the X-direction by a high-speed rotating polygonal mirror. The fluorescent signal emitted by the protein detection reagent is collected by two optical fiber bundles, and specific fluorescent signals are isolated using separate interference filters before being converted to electrical signals by dual photomultiplier tubes. Analog-todigital converters transform the electrical signals into images for data interpretation and analysis. The generated data are proportional to the light signal emitted over a broad linear dynamic range, encompassing four orders of magnitude. Typically, six to eight minutes are required to scan an entire $20 \times$ $25-\mathrm{cm}$ gel. While laser scanners are much slower than fixed CCD camerabased imaging devices, an impressive $50-80-\mu \mathrm{m}$ spatial resolution is achievable compared with the roughly 200$\mu \mathrm{m}$ spatial resolution obtained with standard fixed CCD cameras.

While laser scanners are appropriate for the analysis of gels and electroblots,

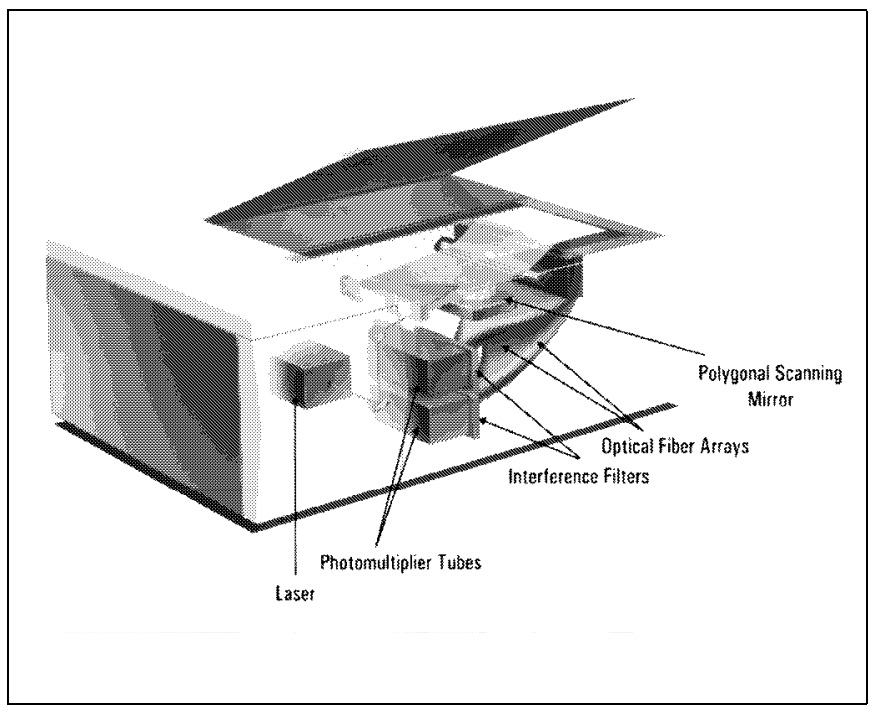

Figure 2. Schematic diagram of the components of a typical laser scanning device. The FMBIO ${ }^{\circledR} \mathrm{II}$ is shown in the diagram, though most other laser systems have similar architecture. The system uses a combination of mechanical and optical scanning to interrogate a gel by 2-D raster scanning. (Diagram courtesy of Dr. William Chapman, Hitachi Software, Alameda, CA, USA.)

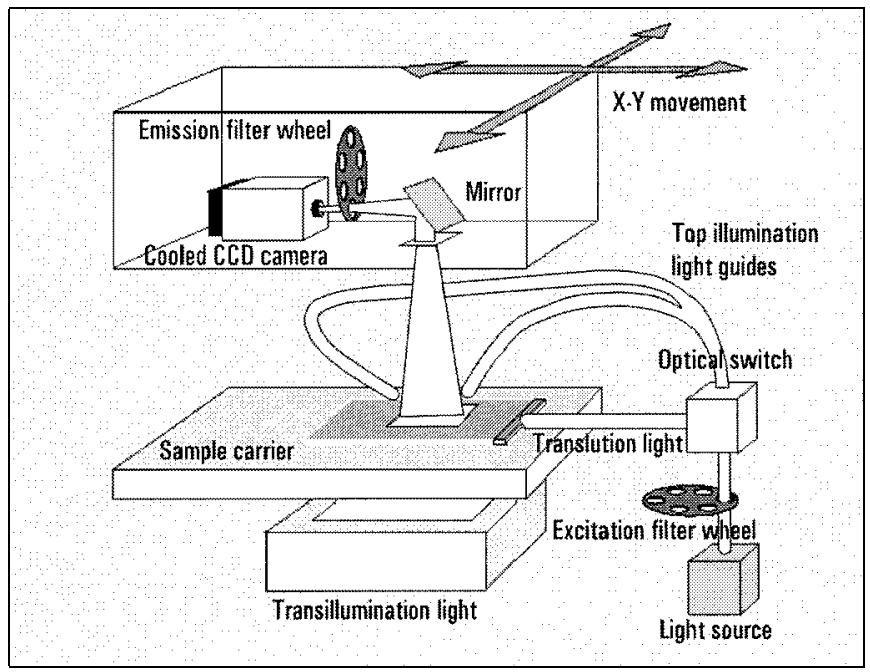

Figure 3. Schematic diagram of the components of a xenon-arc/UV transilluminator, CCD camera-based imaging device. The Perkin-Elmer Arthur ${ }^{\mathrm{TM}} 1442$ Multi-Wavelength Fluorimager is shown in the diagram. This system allows multiple modes of illumination and has multi-wavelength capability provided by the six-position excitation and emission filter wheels. (Illustration courtesy of Dr. Carole Spibey, Perkin-Elmer, Cambridge, UK.) 
they often lack the depth of focus for a convenient analysis of multiwell plates. Some systems, however, do permit the adjustment of the laser's focal point, enabling the use of thicker samples. Black polystyrene 96-well plates for use with certain laser scanners are commercially available and allow highthroughput applications that minimize background fluorescence and light scattering (28). Laser scanners are limited to imaging fluorophores that spectrally match the output of their laser sources. In particular, the systems lack ultraviolet excitation sources.

CCD cameras use silicon chips to convert an image into a sequence of electrical impulses that are stored and subsequently processed (25). Potential wells (pixels) created on the surface of the silicon accumulate charge generated by incoming photons. The physical pixel size on a high-performance CCD camera is about $9 \mu \mathrm{m}$, which equates to about $50 \mu \mathrm{m}$ in the focal plane of the image itself, when a zoom lens is used. After each picture has been taken, the accumulated charges are quickly transferred through the gate electrode to a storage area-the CCD shift register. They then move through an on-chip amplifier to the camera electronics where the signals are converted to a digital format. CCD camera-based image analysis systems often use UV or white light illumination, though highpressure xenon arc sources that provide broadband wavelength coverage are also being introduced. CCD camerabased systems can visualize a wide range of samples including Coomassie Blue, Amido Black, silver, colloidal gold and fluorescently stained gels, as well as autoradiographs, electroblots, thin layer chromatography plates, multiwell plates and microarray biochips.

Cooling the CCD camera reduces background noise about tenfold for every $20^{\circ} \mathrm{C}$ below room temperature. This allows the accumulation of photons on the chip face for extended periods of time without elevating the background signal. On-chip integration allows one to increase the time available for photon capture and provides a high-performance cooled CCD camera with excellent low-light detection capabilities as well as a broad dynamic range (25). This permits the detection of fluorescent bands that are invisible to the eye. Cooled CCD cameras (12-, 14- or 16-bit) are usually used in gel image acquisition and analysis systems. Such systems provide excellent quantitative information over a range of three to four orders of magnitude.

The spatial resolution of currently implemented fixed CCD camera systems is usually inferior to laser scanners and photographic film. For proteomics, large, $20 \times 20-\mathrm{cm}$ gels are usually used, and manually zooming into a particular region of a gel is not practical if a comprehensive mapping effort is to be performed. Thus, operational resolution depends on a combination of the pixel array density and the size of the gel. CCD chips used for gel imaging typically range from $640 \times$ 480 to $1600 \times 1200$ in pixel density, providing an 150-400- $\mu \mathrm{m}$ spatial resolution with the large format 2-D gels typically used in proteomic studies.

Greater resolution is possible by mechanically scanning the CCD camera over the sample and collecting multiple images that are then automatically reconstructed into a complete image of the gel or blot (Figure 3). The main component in such systems is the detector assembly, which consists of the cooled
CCD camera, filter wheel, optics and scanning mechanism. The two-axis scanning mechanism also carries a mirror and the two top illumination light guides, which move with the camera and filter wheel across the image-scanning area. The xenon arc excitation lamp, excitation filter wheel and power supplies are mounted on the bottom panel at the rear frame of the instrument. A UV transilluminator is situated directly beneath the sample carrier. Such systems deliver the same $50-\mu \mathrm{m}$ spatial resolution obtained with laser scanners.

By acquiring images in succession, as many as four different fluorescent labels may be viewed in any one gel. Besides UV transillumination, a xenon arc/CCD camera system is capable of top illumination with the xenon arc light source and edge illumination in translution mode. The latter capability allows edge illumination of gels inside their glass plates, an advantage for low-background detection of prelabeled proteins.

Mechanical scanning CCD systems are relatively slow, with the imaging of large format gels requiring as much as 20 minutes, as opposed to the few seconds required for fixed CCD camera systems. Finally, the integration of multiple images into a composite may

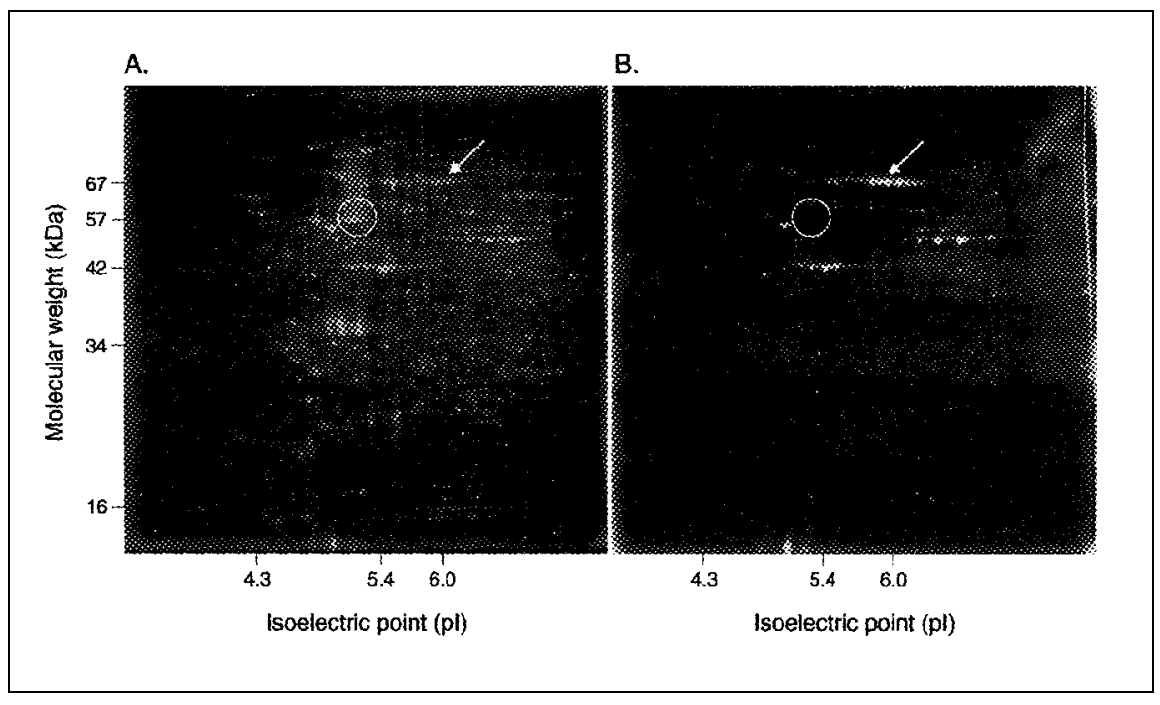

Figure 4. Protein detection by covalent labeling of cysteine residues with monobromobimane compared with noncovalent detection with SYPRO Ruby protein gel stain. (A) Proteins stained with SYPRO Ruby protein gel stain (S-12000; Molecular Probes). (B) Proteins prelabeled with monobromobimane (M-1378; Molecular Probes). While SYPRO Ruby protein gel stain readily reveals the majority of proteins present in fibroblasts, many proteins are not visualized with monobromobimane. Note that serum albumin, present in the media in which the fibroblasts were grown, is strongly labeled (arrows), while vimentin is poorly labeled (circle) with monobromobimane. Serum albumin has 35 , while vimentin has a single cysteine residue. (Figure courtesy of Kiera Berggren, Molecular Probes.) 
generate pictures characterized by a patchwork pattern with varying background intensities in each section. If the gel or blot is not positioned at the proper height, overlap and duplication of data may occur, leading to spot distortion. Manufacturers of such instruments are continually improving their capabilities and minimizing the cited artifacts.

\section{COVALENT DERIVATIZATION METHODS}

The 2-D gel electrophoresis process used for resolving complex samples severely restricts the choice of fluorophores that may be employed to prederivatize proteins. Fluorophores must not significantly perturb protein isoelectric point or molecular weight in this application. To date, only two approaches for covalent pre-derivatization of proteins with fluorophores have successfully been implemented in proteomics studies. Monobromobimane has been used to modify cysteine residues and succinimidyl esters of the unsymmetrical cyanine dyes, propyl-Cy3 and methyl-Cy5, which have been used to modify lysine residues $(14,58,59)$.

Monobromobimane is added directly to solubilized cellular proteins in electrophoresis lysis buffer to obtain maximum fluorescence labeling of sulfhydryl-containing proteins (59). Labeling is rapid, and derivatized proteins are easily visualized as fluorescent turquoise bands on a standard laboratory UV transilluminator. Unfortunately, cysteine residues appear to be especially poor targets for uniform labeling of proteins when compared with methionine or lysine residues (5). Some proteins contain a large amount of cysteine residues such as low-density lipoprotein receptor (61 residues), serum albumin (35 residues), zyxin (21 residues) and vascular endothelial cell growth factor (VEGF; 16 residues), while others are completely devoid of cysteine residues, such as myoglobin, concanavalin $\mathrm{A}$, myelin basic protein, $\beta$-casein, cytochrome b5 and bovine carbonic anhydrase II. Consequently, 2-D protein profiles generated with monobromobimane are significantly different from those produced by other staining techniques (14) (Figure 4). This could, however, be a potential advantage for studies focusing on the characterization of cysteine-rich proteins.

Though monobromobimane labeling is expected to complicate the interpretation of peptide mass profiles by increasing the mass of all derivatized cysteine residues by $190 \mathrm{Da}$, remember that $92 \%$ of the peptides produced from the tryptic digestion of a typical proteome lack cysteine residues and probably will not become derivatized (18). Nonetheless, side reactions with other functional groups such as lysine and histidine residues (as well as the altered specificity of trypsin, arising from its interaction with the chemically generated unnatural amino acid residues) do appear to make interpretation of spectra difficult for highly derivatized proteins (5). In fact, the more extensive the labeling is, the poorer the peptide mass profile results obtained and the more likely the protein will be misidentified by database software (5). At the very least, a corroborating technique such as nanoelectrospray ionization tandem mass spectrometry (NESI-MS/MS) or amino acid compositional analysis must be performed in conjunction with MALDITOF mass spectrometry before attempting to assign an identity to any protein labeled with monobromobimane (5).

Succinimidyl esters of propyl-Cy3 and methyl-Cy5 dyes permit prelabeling of two different protein samples before running them on the same 2-D gel in a type of differential display format (58). Although referred to as $\mathrm{Cy} 3$ and Cy5 dyes, it is important to realize that the dyes used in this difference gel electrophoresis format are significantly more hydrophobic than the popular fluorophores used for antibody labeling. To make the reaction chemistry compatible with 2-D gel electrophoresis, the sulfonate groups of the $\mathrm{Cy}$ dyes were omitted (58). This omission allows regeneration of the positive charge of the lysine residue upon reaction with proteins by replacement with a nonionizable quartenary amino group from the dye. Also, the molecular weights of the two dye molecules are closely matched, though the unreacted proteins in the sample migrate with lower apparent molecular weights.

Covalent attachment of the nonsulfonated $\mathrm{Cy}$ dyes increases protein 
hydrophobicity. As a result, overall protein solubility is substantially reduced after derivatization with these dyes. Natively hydrophobic proteins are conspicuously absent from current 2-D gel profiles, and it is likely that other proteins are lost from the patterns after their derivatization with the hydrophobic dyes (63). Because of the profound impact of the hydrophobic dyes on solubility, it is necessary to derivatize a dwindling number of proteins in a sample (on average $1 \%-2 \%$ of the lysine residues) and detect them with a sensitive, cooled CCD camera (58). Despite the low degree of substitution, the modified proteins migrate to a different position than the unmodified proteins in the gel. Because of the differences in mobility, regions of gel that are biased by about one spot diameter to a lower molecular weight must be excised to obtain material for analysis by mass spectrometry or Edman sequencing. In essence, one is guessing where the unmodified protein migrates, which increases the possibility that the wrong protein will be characterized. Also, the effective resolution of the 2-D gel is reduced by half because of the duplication of protein spots in the molecular weight dimension. The propyl Cy3/methyl Cy5 procedure is fully compatible with amino acid analysis, Edman sequencing and peptide mass profiling because of the small number of amino acid residues actually derivatized by the method.

\section{NONCOVALENT DETECTION METHODS}

Nile Red dye (9-diethylamino-5Hbenzo[a]phenoxazine-5-one), $\mathrm{SYPRO}^{\circledR}$ Red dye, SYPRO Orange dye and SYPRO Tangerine dye interact with proteins noncovalently through association with SDS-protein complexes $(1,2,7,12,13,53-55)$. Since SDS binds to protein with a fairly constant stoichiometry, protein quantitation based on an interaction with the detergent should be more reliable than one based on interactions with primary amines alone. Besides the dyes that intercalate into SDS micelles, SYPRO Ruby and SYPRO Rose dyes are stains that bind to proteins by an electrostatic mechanism similar to Coomassie Blue stain

Table 1. Fluorescent Protein Reporters with Bimodal Excitation Spectra

\begin{tabular}{|lccc|}
\hline Dye & $\begin{array}{c}\text { UV } \\
\text { excitation } \\
(\mathbf{n m})\end{array}$ & $\begin{array}{c}\text { Visible light } \\
\text { excitation } \\
\mathbf{( n m})\end{array}$ & $\begin{array}{c}\text { Emission } \\
\text { maximum } \\
\mathbf{( n m )}\end{array}$ \\
\hline Nile Red Dye & 300 & 540 & 640 \\
SYPRO Orange Dye & 300 & 470 & 570 \\
SYPRO Red Dye & 300 & 550 & 630 \\
SYPRO Ruby Dye & 280 & 450 & 610 \\
SYPRO Tangerine Dye & 300 & 490 & 640 \\
\hline
\end{tabular}

$(5,6,31,44,52)$. These luminescent transition metal complexes allow sensitive fluorescent detection of proteins after isoelectric focusing gel electrophoresis, SDS-PAGE, 2-D gel electrophoresis and electroblotting $(5,6,31,44,52)$.

The fluorescent oxazone dye, Nile Red is compatible with standard Edman sequencing and immunodetection procedures, but a detection sensitivity of 5-50 ng for proteins in SDS polyacrylamide gels is only slightly better than detection obtained with Coomassie Blue staining $(1,2,7,12,13,53-55)$. Nile Red dye is nearly insoluble in water, and stock solutions are prepared in dimethyl sulfoxide (13). Staining of proteins in SDS polyacrylamide gels is usually performed in a solution of $2 \%$ dimethyl sulfoxide in water (7). Consequently, the Nile Red staining procedure permits transfer of proteins to PVDF membranes by electroblotting after staining, unlike fixative-containing stains such as Coomassie Blue and silver stain $(2,7)$. Unfortunately, the Nile Red dye staining method is plagued with problems of nonspecific precipitation on the surfaces of gels and in the staining tray. Also, significant photobleaching of the dye is observed even after brief exposure to UV illumination (13).

SYPRO Red, SYPRO Orange and SYPRO Tangerine dyes detect proteins in SDS polyacrylamide gels using an easy, one-step staining procedure that requires 30-60 minutes to complete and does not involve a destaining step (53-55). As with Nile Red dye, staining is achieved through intercalation with SDS/protein micelles. Although hydrophobic in nature, these SYPRO dyes are more water soluble and do not suffer from problems of nonspecific pre- cipitation on gel surfaces and staining trays, though relatively minor speckling of fluorescent stains may be observed.

In contrast to many silver staining methods, the SYPRO dyes do not significantly stain nucleic acids or bacterial lipopolysaccharides $(53,55)$. Four to ten nanograms of protein can routinely be detected with this group of SYPRO dyes, rivaling the sensitivity of rapid silver staining techniques and surpassing the best colloidal Coomassie Blue staining methods available (53-55). Both SYPRO Red and SYPRO Orange dyes require $7 \%$ acetic acid in the staining solution, which is problematic when experiments require electroblotting, electroeluting or measuring enzyme activity.

If acetic acid is omitted from the staining solution, proteins may be recovered from gels, but the detection sensitivity obtained with SYPRO Red and SYPRO Orange stains is considerably lower, and significant protein-to-protein staining variability is observed (53). SYPRO Tangerine stain does not require solvents such as methanol or acetic acid for effective protein visualization (55). Instead, proteins can be stained in a wide range of buffers, including PBS or simply $150 \mathrm{mM} \mathrm{NaCl}$. Since staining using SYPRO Tangerine dye does not require harsh fixatives, proteins are easily eluted from gels or used in zymographic assays, when SDS, which is present during electrophoresis, does not inactivate them (55). The dye is also suitable for staining proteins in gels before their transfer to membranes by electroblotting. Using a fixative, 7\% acetic acid staining solution, SYPRO Red and SYPRO Orange dyes perform better than the SYPRO Tangerine dye, as the latter dye leads to slightly higher 
Table 2. Comparisons of Several Fluorescence Detection Methods for Proteomic Studies

\begin{tabular}{|c|c|c|c|}
\hline Parameter & Monobromobimane & Propyl-Cy3/Methyl-Cy5 & SYPRO Ruby dye \\
\hline Electrophoretic separation & $\begin{array}{l}\text { Increases protein hydrophobicity } \\
\text { somewhat, causing protein } \\
\text { "comet tails". }\end{array}$ & $\begin{array}{l}\text { Increases protein } \\
\text { hydrophobicity, } \\
\text { alters protein } \\
\text { migration somewhat } \\
\text { relative to unlabeled } \\
\text { protein. }\end{array}$ & $\begin{array}{l}\text { Post-electrophoresis } \\
\text { stain, so does not } \\
\text { alter protein } \\
\text { migration. }\end{array}$ \\
\hline Labeling/staining & $\begin{array}{l}\text { Labeling performed before } \\
\text { electrophoresis. }\end{array}$ & $\begin{array}{l}\text { Labeling performed } \\
\text { before electrophoresis. }\end{array}$ & $\begin{array}{l}1-2 \text { steps required } \\
\text { after electrophoresis. }\end{array}$ \\
\hline Limits of detection & $4-30 \mathrm{ng}$ & $1-2 \mathrm{ng}$ & $1-2 \mathrm{ng}$ \\
\hline Linear dynamic range & 2 orders of magnitude & 3 orders of magnitude & 3 orders of magnitude \\
\hline $\begin{array}{l}\text { Moles fluorophore bound/ } \\
\text { mole protein }\end{array}$ & $\begin{array}{l}\text { Depends on number of cysteine } \\
\text { residues. Typically 0-90 residues. } \\
\text { Poor correlation with } \\
\text { molecular weight. }\end{array}$ & $\begin{array}{l}0.04-0.1 \text { ( } 1 \text { in } 100 \text { lysine } \\
\text { residues are labeled and } \\
\text { proteins are typically } \\
\text { composed of } 4 \%-10 \% \\
\text { lysine residues). }\end{array}$ & $\begin{array}{l}\text { Depends on number } \\
\text { of lysine, arginine } \\
\text { and histidine } \\
\text { residues. Typically } \\
4-400 \text { residues. } \\
\text { Good correlation } \\
\text { with molecular weight. }\end{array}$ \\
\hline Molar extinction coefficient & $5000 \mathrm{~cm}^{-1} \mathrm{M}^{-1}$ & $150000 \mathrm{~cm}^{-1} \mathrm{M}^{-1}$ & $30000 \mathrm{~cm}^{-1} \mathrm{M}^{-1}$ \\
\hline $\begin{array}{l}\text { Protein-to protein } \\
\text { variation in detection. }\end{array}$ & $\begin{array}{l}\text { Very high. Number of cysteine } \\
\text { residues varies significantly from } \\
\text { protein to protein. }\end{array}$ & $\begin{array}{l}\text { Substoichiometric } \\
\text { method that labels } \\
\text { abundant proteins } \\
\text { with high lysine } \\
\text { content preferentially. }\end{array}$ & $\begin{array}{l}\text { Modest (similar to } \\
\text { colloidal Coomassie } \\
\text { Blue dye). }\end{array}$ \\
\hline $\begin{array}{l}\text { Compatibility with } \\
\text { commercially available } \\
\text { laser scanners. }\end{array}$ & Not compatible. & $\begin{array}{l}\text { Restricted to specialized } \\
\text { dual wavelength systems. } \\
\text { (532-nm SHG or YAG } \\
\text { combined with 633-nm } \\
\text { He-neon or 635-nm } \\
\text { diode laser). }\end{array}$ & $\begin{array}{l}\text { Compatible with } \\
\text { 473-nm } \\
\text { SHG, 488-nm Ar, } \\
\text { 532-nm SHG, } \\
\text { 532-nm YAG lasers. } \\
\text { Not compatible } \\
\text { with 633-nm } \\
\text { He-Ne or 635-nm } \\
\text { diode lasers. }\end{array}$ \\
\hline $\begin{array}{l}\text { Compatibility with } \\
\text { CCD/UV systems. }\end{array}$ & $\begin{array}{l}\text { Compatible with common } \\
\text { UV-light sources. }\end{array}$ & Not compatible. & $\begin{array}{l}\text { Compatible with } \\
\text { common UV } \\
\text { light sources. }\end{array}$ \\
\hline $\begin{array}{l}\text { Compatibility with CCD/ } \\
\text { xenon-arc systems }\end{array}$ & Compatible. & Compatible. & Compatible. \\
\hline $\begin{array}{l}\text { Amino acid sequencing } \\
\text { by Edman degradation. }\end{array}$ & Unknown. & $\begin{array}{l}\text { Good compatibility, except } \\
\text { for possibility of spot } \\
\text { registration errors. }\end{array}$ & Good compatibility. \\
\hline $\begin{array}{l}\text { Peptide mass profiling by } \\
\text { MALDI-TOF mass } \\
\text { spectrometry. }\end{array}$ & Poor compatibility. & $\begin{array}{l}\text { Good compatibility, } \\
\text { except for possibility } \\
\text { of spot registration errors. }\end{array}$ & Good compatibility. \\
\hline
\end{tabular}


nonspecific background staining (55). All three fluorescent dyes are fully compatible with Edman-based sequencing and mass spectrometry $(19,20,55)$.

These three dyes are not recommended for detection of proteins electroblotted to membranes, separated by isoelectric focusing or fractionated by nondenaturing gel electrophoresis. Also, their detection sensitivity in 2-D gels is somewhat poorer than in simple, one-dimensional SDS polyacrylamide gels. This appears to be due to the mixed micelles that contain nonionic detergents such as Triton ${ }^{\circledR} \mathrm{X}-100$ as well as carrier ampholytes. For 2-D gels, high-quality silver staining procedures usually surpass these fluorescent dyes in terms of detection sensitivity, while colloidal Coomassie Blue offers a comparable level of sensitivity.

SYPRO Ruby and SYPRO Rose stains were developed specifically for broad compatibility with commonly used microchemical characterization protocols. They do not contain extraneous chemicals such as formaldehyde, glutaraldehyde, or Tween ${ }^{\circledR} 20$ that are known to compromise such procedures. These luminescent transition metal chelate stains are easily incorporated into integrated proteomics platforms that combine automated gel stainers, image analysis workstations, robotic spot excision instruments, protein digestion workstations and mass spectrometers (32-34). The most sensitive luminescent stain, SYPRO Ruby stain, is more sensitive and exhibits a broader linear dynamic range than the best silver staining procedures available.

SYPRO Rose stain is useful for reversibly detecting proteins on nitrocellulose or PVDF membranes $(31,44)$. It is readily removed from proteins by incubating at neutral to mildly alkaline pH. Compatibility with immunoblotting, lectin blotting, Edman sequencing and mass spectrometry make SYPRO Rose stain a good routine protein detection reagent that is nearly as simple to use as Ponceau S stain (31). As originally formulated, SYPRO Rose stain exhibits intense blue fluorescence at $450 \mathrm{~nm}$ because of the uncomplexed ligand and the desired red fluorescence at 595 and $615 \mathrm{~nm}$. Incorporating a thermodynamically more stable ligand has resulted in a much brighter stain, referred to as SYPRO Rose Plus Protein Blot Stain, that has a single, narrow emission peak at $615 \mathrm{~nm}$.

SYPRO Ruby dye is an optimized ruthenium-based metal chelate stain developed to address the limitations of the other SYPRO dyes. SYPRO Ruby Protein Blot stain permanently stains electroblotted proteins on nitrocellulose and PVDF membranes with a detection sensitivity of $0.25-1 \mathrm{ng}$ protein $/ \mathrm{mm}^{2}$ in slot-blotting applications. This translates to between 2 and $8 \mathrm{ng}$ protein in routine electroblotting applications (6). Side-by-side comparisons demonstrate that SYPRO Ruby Protein Blot stain is as sensitive as colloidal gold stain but is vastly superior in providing a linear dynamic range that extends over three logs of protein concentration (6). Unlike colloidal gold stain, SYPRO Ruby stain does not interfere with mass spectrometry, Edman-based sequencing or immunodetection procedures (6).

SYPRO Ruby Protein Gel stain and SYPRO Ruby IEF Protein Gel stain allow one-step, low-background staining of proteins in gels without resorting to lengthy destaining steps. The linear dynamic range of these dyes extends over three orders of magnitude and surpasses silver and Coomassie blue stains in performance. Proteins that stain poorly with silver stain techniques are often readily detected by SYPRO Ruby dye $(5,52)$. Although they are more sensitive than SYPRO Orange, Red and Tangerine dyes, optimal staining is somewhat slower, requiring about four hours to complete. Similar to colloidal Coomassie Blue stain but unlike silver stain, SYPRO Ruby dye stains are endpoint stains. Thus, staining times are not critical, and staining can be performed over night without gels overdeveloping. SYPRO Ruby Protein Gel stain and SYPRO Ruby IEF Protein Gel stain are ideally suited for use in the identification of proteins by peptide mass profiling using MALDI-TOF mass spectrometry $(5,52)$.

\section{ADVANTAGES OF DYES WITH BI- MODAL EXCITATION SPECTRA}

The automation of proteomics is still a work in progress. Laboratories engaged in global protein analysis dif- fer substantially in the degree of robotic systems used for high-throughput characterization of proteins. Though automated workstation environments for proteome analysis are becoming more commonplace in agricultural, biotechnology and pharmaceutical environments, most academic laboratories are still performing most of these tasks manually and submitting excised spots to institutional core facilities for protein identification. Fluorescent spot-picking robots are being developed by at least two commercial manufacturers and may be available by the summer of 2000. Typically, academic institutions own a single analytical imaging system that was purchased with a shared resource equipment grant. For broadest compatibility, when using optimized fluorescent dyes developed for proteomics applications, one must consider the requirement of laser scanners for visible light excitation and of common CCD camera systems and laboratory equipment such as transilluminators for UV excitation.

The covalent protein adducts resulting from alkylation of cysteine residues by monobromobimane fluoresce with an excitation maximum of $385 \mathrm{~nm}$ and an emission maximum of about $470 \mathrm{~nm}$ (59). After 2-D gel electrophoresis, proteins are readily imaged using a 365 nm UV transilluminator or a xenon arc light source combined with appropriate excitation and emission filters. However, the dye is not readily detected by commercial laser scanning devices. Similarly, a limitation of both SYPRO Rose and SYPRO Rose Plus dyes is that they can only be visualized on systems using UV epi-illumination or xenon arc lamp sources equipped with a $390 \pm 35 \mathrm{~nm}$ excitation filter $(31,44)$.

Methyl-Cy5-derivatized proteins maximally excite at $650 \mathrm{~nm}$ and maximally emit at $670 \mathrm{~nm}$, while propylCy3-derivatized proteins maximally excite at about $550 \mathrm{~nm}$ and maximally emit at $570 \mathrm{~nm}$. Consequently, their use is restricted to several specialized instruments such as those that use CCD cameras combined with xenon arc sources and those using specialized dual laser sources. The dye pair cannot be visualized effectively by simply placing a gel on a UV transilluminator. This is problematic for mass spectrom- 
etry studies that involve direct manual excision of protein spots from the gels, as the primary commercial instruments for detecting the dyes do not allow the user to directly view the gel.

A homemade viewing system may be built using 100-W halogen lamps and appropriate filters, but the protein/cyanine dye conjugates are so dim that a 30 -min camera exposure time is needed to view $1 \mathrm{ng}$ protein (58). Only the most abundant proteins in a sample may readily be seen. Manual excision of spots from propyl-Cy3/ methyl-Cy5labeled protein patterns is probably best accomplished indirectly as is often done with autoradiographs. The gel should be placed on a one-to-one size ratio printout of the fluorescent image produced from an appropriate gel documentation system and spots excised with reference to the printout.

Commercially available fluorescent protein gel stains that display bimodal excitation spectra are listed in Table 1. Nile Red and SYPRO Red dyes may be imaged using a 302-nm UV transilluminator, xenon arc source, 532-nm SHG or 532-nm Nd-YAG laser. SYPRO Orange, SYPRO Tangerine and SYPRO Ruby gel stains may be visualized using a wide range of excitation sources commonly used in image analysis systems, including a UV transilluminator, 473-nm SHG laser, 488$\mathrm{nm}$ argon-ion laser, 532-nm Nd-YAG laser, 532-nm SHG laser, xenon arc lamp, 460-nm blue fluorescent light bulb or 470-nm blue light-emitting diode (LED) $(5,6,52-56)$.

Since all the dyes excite at roughly $300 \mathrm{~nm}$, the excision of spots from stained gels can readily be accomplished on a standard laboratory UV transilluminator. In addition, visible blue light illumination sources that are appropriate for the detection of these fluorophores have been developed by many manufacturers (5-6,52-56). Blue light sources are less hazardous than UV transilluminators and minimize photobleaching of fluorophores. The fluorescent dyes may be quantified with commercially available CCD camera-based image analysis workstations or a variety of laser scanners, providing a linear dynamic range of three orders of magnitude. They are also readily documented using simple photographic equipment.
SYPRO Ruby and SYPRO Rose dyes contain the luminescent transition metals ruthenium and europium. They may be used for time-resolved imaging of gels and blots in the near future, which will increase detection sensitivity by as much as two to three orders of magnitude relative to the prompt detection of the stains currently performed (49). If such performance improvements are achieved, these fluorescent stains should be able to detect low picogram instead of low nanogram amounts of protein. Instrumentation capable of time-resolved imaging is just being commercially introduced, and its further refinement and multiplication are all but assured as image-based, high-throughput screening assays are implemented in the drug discovery industry.

\section{CONCLUSION}

In terms of automation, research methods for parallel characterization of proteins have trailed DNA- and mRNAbased approaches because of the complexity of the technology required to separate, analyze and identify the thousands of different polypeptides encoded by a typical genome $(37,50)$. Because DNA and mRNA are chemically quite homogeneous, the mechanization of genomic techniques such as DNA sequencing, mRNA isolation, cloning and PCR amplification is relatively straightforward, and the methods are conveniently applied to any gene with little modification. Proteins, on the other hand, are chemically quite heterogeneous because of their diverse functional roles in cells, and as a result techniques appropriate for one protein are often unsuitable for others. Particularly difficult to automate are 2-D gel electrophoresis and image analysis, two labor-intensive tasks (32-34). However, automation and integration of the individual steps required for proteomics are key to increasing process throughput and facilitating target identification in global protein studies.

Fluorescence detection technologies will likely play a critical role in streamlining the proteomics process. Three different fluorescence detection strategies are summarized in Table 2 with respect to their applicability to semiautomated 
proteomics environments. Current massspectrometry-based methods require very low nanogram levels of protein detection. Though silver staining procedures detect proteins in the low nanogram range, they are cumbersome, multistep processes that exhibit poor linear dynamic responses. Compatibility with mass spectrometry requires the omission of glutaraldehyde from silver stain formulations, which significantly compromises staining performance (51). An additional destaining step is also recommended to reduce background interference and suppression of signals often encountered in peptide mass profiling experiments (15). Procedures for implementing fluorescence detection are technically much simpler than silver staining procedures, quantitative information is more readily obtained and compatibility with downstream microchemical methods is generally better.

The degree that automated proteomics tasks have been put to use in the research environment varies consid- erably from laboratory to laboratory, with many facilities performing the entire process manually by using systems assembled from various unrelated components that have not been specifically designed for the proficient handling of samples and data throughout the entire process. Fluorescent dyes with bimodal excitation spectra such as Nile Red, SYPRO Red, SYPRO Orange and SYPRO Ruby protein gel stains are compatible with a broad spectrum of instrumentation that are already available at most institutions, from simple UV light boxes to CCD camera- and laser-based gel scanners.

\section{ACKNOWLEDGMENTS}

The author is grateful to Tom Steinberg, Kiera Berggren, Courtenay Kemper, Jill Hendrickson, Vicki Singer and Richard Haugland of Molecular Probes, Inc. for intellectual contributions to this project. Their input was critical to the success of the research. The author is also grateful to Mary Lopez and Elena Chernokalskaya of Genomic Solutions for valuable contributions to the project in the fields of mass spectrometry and automation. The author thanks Carole Spibey of Perkin-Elmer and William Chapman of Hitachi Software for providing information about CCD-based and laserbased imaging systems.

\section{REFERENCES}

1.Alba, F., A. Bermudez, S. Bartolome and J. Daban. 1996. Detection of five nanograms of protein by two-minute Nile red staining of unfixed SDS gels. BioTechniques 21:625-626.

2.Alba, F. and J. Daban. 1998. Rapid fluorescent monitoring of total protein patterns on sodium dodecyl sulfate-polyacrylamide gels and western blots before immunodetection and sequencing. Electrophoresis 19:2407-2411.

3.Allen, R. and B. Budowle. 1999. Protein Staining and Identification Techniques. BioTechniques Molecular Laboratory Methods Series, Eaton Publishing, Natick, MA.

4.Anderson, L. and J. Seilhamer. 1997. A comparison of selected mRNA and protein abundances in human liver. Electrophoresis 18:533537.

5.Berggren, K., T. Steinberg, C. Kemper, M. Lopez, C. Chernokalskaya, Z. Diwu, R. Haugland and W. Patton. Background-free, high-sensitivity staining of proteins in one- and two-dimensional sodium dodecyl sulfate-poly- acrylamide gels using a luminescent ruthenium complex. Electrophoresis (In Press).

6.Berggren, K., T. Steinberg, W. Lauber, J. Carroll, M. Lopez, E. Chernokalskaya, L. Zieske, Z. Diwu, R. Haugland and W. Patton. 1999. A luminescent ruthenium complex for ultrasensitive detection of proteins immobilized on membrane supports. Anal. Biochem. 272:129-143.

7.Bermudez, A., J. Daban, J. Garcia and E. Mendez. 1994. Direct blotting, sequencing and immunodetection of proteins after five-minute staining of SDS and SDS-treated IEF gels with Nile Red. BioTechniques 16:621-624.

8.Berndt, P., U. Hobohm and H. Langen. 1999. Reliable automatic protein identification from matrix-assisted laser desorption/ionization mass spectrometric peptide fingerprints. Electrophoresis 20:3521-3526.

9.Blomberg, A., L. Blomberg, J. Norbeck, S. Fey, P. Mose-Larsen, M. Larsen, P. Roepstorff, H. Degand, M. Boutry et al. 1995. Interlaboratory reproducibility of yeast protein patterns analyzed by immobilized $\mathrm{pH}$ gradient two-dimensional gel electrophoresis. Electrophoresis 16:1935-1945.

10.Boulikas, T. 1995. Phosphorylation of transcription factors and control of the cell cycle. Crit. Rev. Eukaryot. Gene Expr. 5:1-77.

11.Brush, M. 1998. Dye hard: protein gel staining products. The Scientist 12:16-22.

12.Daban, J., S. Bartolome and A. Bermudez. 1996. Rapid staining of proteins in polyacrylamide gels, p. 179-185. In J. Walker (Ed.), The Protein Protocols Handbook. Humana Press, Totowa, NJ.

13.Daban, J., S. Bartolome and M. Samso. 1991. Use of the hydrophobic probe Nile Red for the fluorescent staining of protein bands in sodium dodecyl sulfate-polyacrylamide gels. Anal. Biochem. 199:169-174.

14.Fey, S., A. Nawrocki, M. Larsen, A. Gorg, P. Roepstorff, G. Skews, R. Williams and P. Larsen. 1997. Proteome analysis of Saccharomyces cerevisiae: a methodological outline. Electrophoresis 18:1361-1372.

15.Gharahdaghi, F., C. Weinberg, D. Meagher, B. Imai and S. Mische. 1999. Mass spectrometric identification of proteins from silverstained polyacrylamide gel: a method for the removal of silver ions to enhance sensitivity. Electrophoresis 20:601-605.

16.Gwynne, P. and G. Page. 1999. Protein + protein interactions. Science 286:1759-1773.

17.Gygi, S., B. Rist, S. Gerber, F. Turecek, M. Gelb and R. Aebersold. 1999. Quantitative analysis of complex protein mixtures using isotope-coded affinity tags. Nat. Biotechnol. 17:994-999.

18.Gygi, S., Y. Rochon, B. Franza and R. Aebersold. 1999. Correlation between protein and mRNA abundance in yeast. Mol. Cell. Biol. 19:1720-1730.

19.Hamby, R. 1996. A sensitive fluorescent gel stain. Am. Biotechnol. Lab. 14:12.

20.Hamby, R. 1997. A fluorescent gel stain for detection of proteins following 1-D, 2-D and native polyacrylamide gel electrophoresis. Biotechnol. Int. 1:339-343.

21.Hardie, D. 1990. Roles of protein kinases and phosphatases in signal transduction. Symp. Soc. Exp. Biol. 44:241-255. 
22.Hastie, L., W. Patton, H. Hechtman and D. Shepro. 1997. $\mathrm{H}_{2} \mathrm{O}_{2}$-induced filamin redistribution in endothelial cells is modulated by the cyclic AMP dependent protein kinase pathway. J. Cell. Physiol. 172:373-381.

23.Hastie, L., W. Patton, H. Hechtman and D. Shepro. 1997. Hydrogen peroxide-induced filamin translocation in an in vitro model of reoxygenation injury; inhibition by iron chelators and superoxide radical scavengers. Free Radic. Biol. Med. 22:955-966

24.Hastie, L., W. Patton, H. Hechtman and D. Shepro. 1998. Metabolites of the phospholipase $D$ pathway regulate $\mathrm{H}_{2} \mathrm{O}_{2}$-induced filamin redistribution in endothelial cells. J. Cell. Biochem. 68:511-524.

25.Holton, W. 1994. CCD technology has brought profound changes to the world of bioimaging. BioPhotonics 1:43-51.

26.Hucho, F. and K. Buchner. 1997. Signal transduction and protein kinases: the long way from the plasma membrane into the nucleus. Naturwissenschaften 84:281-290.

27.Humphrey-Smith, I. and W. Blackstock. 1997. Proteome analysis: genomics via the output rather than the input code. J. Protein Chem. 16:537-544.

28.Johnson, B. 1999. All's well that ends well; a profile of specialty microwell plates. The Scientist 13:16.

29.Karin, M. 1994. Signal transduction from the cell surface to the nucleus through the phosphorylation of transcription factors. Curr. Opin. Cell. Biol. 6:415-424.

30.Krause, E., H. Wenschuh and P. Jungblut. 1999. The dominance of arginine-containing peptides in MALDI-derived tryptic mass fingerprints of proteins. Anal. Chem. 71:41604165

31.Lim, M., W. Patton, N. Shojaee, M. Lopez, K. Spofford and D. Shepro. 1997. A luminescent europium complex for the sensitive detection of proteins and nucleic acids immobilized on membrane supports. Anal. Biochem. 245: 184-195.

32.Lopez, M. 1998. Proteomic databases: roadmaps for drug discovery. Am. Biotechnol. Lab. 16:8-9.

33.Lopez, M. 1999. Automating proteome analysis: from soup to nuts. Am. Biotechnol. Lab. 17:44-45.

34.Lopez, M. 1999 Proteome analysis I. Gene products are where the biological action is. J. Chromatogr. B 722:191-202.

35.Lopez, M. and W. Patton. 1997. Reproducibility of polypeptide spot positions in two-dimensional gels run using carrier ampholytes in the isoelectric focusing dimension. Electrophoresis 18:338-343.

36.Mullner, S., T. Neumann and F. Lottspeich. 1998. Proteomics - a new way for drug target discovery. Arzneimittelforschung 48:93-95.

37.Nowak, R. 1995. Entering the postgenome era. Science 270:368-369.

38.Oda, Y., K. Huang, F. Cross, D. Cowburn and B. Chait. 1999. Accurate quantitation of protein expression and site-specific phosphorylation. Proc. Natl. Acad. Sci. USA 96:65916596.

39.O'Farrell, P. 1975. High resolution two-dimensional electrophoresis of proteins. J. Biol. Chem. 250:4007-4021.
40.Patton, W. 1995. Biologist's perspective on analytical imaging systems as applied to protein gel electrophoresis. J. Chromatogr. A 698:5587.

41.Patton, W. 1999. Proteome analysis II. Protein subcellular redistribution: linking physiology to genomics via the proteome and separation technologies involved. J. Chromatogr. B 722:203223.

42.Patton, W., H. Erdjument-Bromage, A. Marks, M. Taubman and P. Tempst. 1995. Components of the protein synthesis and folding machinery are induced in both hyperplastic and hypertrophic vascular smooth muscle cells. Identification by comparative protein phenotyping and micro-sequencing. J. Biol. Chem. 270:21404-21410.

43.Patton, W., M. Lim and D. Shepro. 1999. Image acquisition in 2-D electrophoresis, p. 353362. In A. Link (Ed.), Methods in Molecular Biology, Volume 112. 2-D Proteome Analysis Protocols. Humana Press, Totowa, NJ.

44.Patton, W., M. Lim and D. Shepro. 1999. Protein detection using reversible metal chelate stains. p. 331-339. In A. Link (Ed.), Methods in Molecular Biology, Volume 112. 2-D Proteome Analysis Protocols. Humana Press, Totowa, NJ

45.Patton, W., M. Pluskal, W. Skea, J. Buecker, M. Lopez, R. Zimmerman, L. Belanger and P. Hatch. 1990. Development of a dedicated two-dimensional gel electrophoresis system that provides optimal reproducibility and polypeptide resolution. BioTechniques 8:518527.

46.Quadroni, M. and P. James. 1999. Proteomics and automation. Electrophoresis 20:664-677.

47.Rabilloud, T. 1999. Silver staining of 2-D electrophoresis gels, p. 297-305. In A. Link (Ed.), Methods in Molecular Biology, Volume 112, 2 D Proteome Analysis Protocols. Humana Press, Totowa, NJ.

48.Rabilloud, T. 2000. Detecting proteins separated by 2-D gel electrophoresis. Anal. Chem. 72:48A-55A

49.Ramm, P. 1999. Imaging systems in assay screening. Drug Discovery Today 4:401-410.

50.Scangos, G. 1997. Drug discovery in the postgenomic era. Nat. Biotechnol. 15:12201221.

51.Shevchenko, A., M. Wilm, O. Vorm and M. Mann. 1996. Mass spectrometric sequencing of proteins silver-stained polyacrylamide gels. Anal. Chem. 68:850-858

52.Steinberg, T., E. Chernokalskaya, K. Berggren, M. Lopez, Z. Diwu, R. Haugland and W. Patton. 2000. Ultrasensitive fluorescence protein detection in isoelectric focusing gels using a ruthenium metal chelate stain. Electrophoresis 21:486-496.

53.Steinberg, T., R. Haugland and V. Singer. 1996 Applications of SYPRO Orange and SYPRO Red protein gel stains. Anal. Biochem. 239:238-245

54.Steinberg, T., L. Jones, R. Haugland and V. Singer. 1996. SYPRO Orange and SYPRO Red protein gel stains: one-step fluorescent staining of denaturing gels for detection of nanogram levels of protein. Anal. Biochem. 239:223-237.

55.Steinberg, T., W. Lauber, K. Berggren, C. Kemper, S. Yue and W. Patton. 2000. Fluo- rescence detection of proteins in SDS-polyacrylamide gels using environmentally benign, non-fixative, saline solution. Electrophoresis 21:497-508.

56.Steinberg, T., H. White and V. Singer. 1997. Optimal filter combinations for photographing SYPRO Orange or SYPRO Red dye-stained gels. Anal. Biochem. 248:168-172.

57.Sutherland, J. 1993. Electronic imaging of electrophoretic gels and blots, p. 1-42. In A. Chrambach, M. Dunn and B. Radola (Eds.), Advances in Electrophoresis, Volume 6. VCH Publishers, New York.

58.Unlu, M., M. Morgan and J. Minden. 1997. Difference gel electrophoresis: a single gel method for detecting changes in protein extracts. Electrophoresis 18:2071-2077.

59.Urwin, V. and P. Jackson. 1993. Two-dimensional polyacrylamide gel electrophoresis of proteins labeled with the fluorophore monobromobimane prior to first-dimensional isoelectric focusing: imaging of the fluorescent protein spot patterns using a cooled charge-coupled device. Anal. Biochem. 209:57-62.

60.Wang, Q., W. Patton, E. Chiang, H. Hechtman and D. Shepro. 1996. Bradykinin-induced filamin translocation in endothelial cells; regulation by calcium, protein kinases and protein phosphatases. J. Cell. Biochem. 62:383396.

61.Wang, Q., W. Patton, H. Hechtman and D. Shepro. 1997. A novel, anti-inflammatory peptide inhibits endothelial cell cytoskeletal rearrangement, nitric oxide synthase translocation and paracellular permeability increases. J. Cell. Physiol. 172:171-182.

62.Wang, Q., W. Patton, H. Hechtman and D. Shepro. 1997. Activation of endothelial cell kinin receptors leads to intracellular calcium increases and filamin translocation: regulation by protein kinase C. Cell. Signal. 9:595-602.

63.Wilkins, M., E. Gasteiger, J. Sanchez, A. Bairoch and D. Hochstrasser. 1998. Two-dimensional gel electrophoresis for proteome projects: the effects of protein hydrophobicity and copy number. Electrophoresis 19:15011505

64.Wirth, P. and A. Romano. 1995. Staining methods in gel electrophoresis, including the use of multiple detection methods. J. Chromatogr. A. 698:123-143.

65.Woods, A., A. Huang, R. Cotter, G. Pasternack, D. Pardoll and E. Jaffee. 1995. Simplified high sensitivity sequencing of MHC class I-associated, immunoreactive peptides using matrix assisted laser desorption/ionization mass spectrometry. Anal. Biochem. 226:15-25.

Address correspondence to:

Dr. Wayne F. Patton

Bioanalytical Assay Development Group

Molecular Probes, Inc.

4849 Pitchford Avenue

Eugene, OR 97402, USA

Internet: waynepatton@probes.com 